MATEC Web of Conferences 11,01027 (2014)

DOI: $10.1051 /$ matecconf / 20141101027

(C) Owned by the authors, published by EDP Sciences, 2014

\title{
Use of metallic fibers in concretes
}

\author{
Souad KHERBACHE, A/Kader TAHAKOURT and Karim MOUSSACEB \\ University of A/MIRA, Department de civil engineering, Bejaia, Algeria \\ Laboratory Engineering Building and Architecture
}

\begin{abstract}
The addition of a waste (fibers) in construction materials, particularly, the concretes is a technique increasingly used, for several reasons, either ecological, or economic, or to improve some properties in a fresh or hardened state. In our work we studied the behavior of the concrete and the mortar containing metallic fibers resulting from the unit BCR which is in Bordj-Menaiel in Algeria (metallic fibers resulting from the rejection at the end of the domestic operation of silvering of the tools and which is stored in plastic bags which are preserved in metal containers). Our work consists to study the behavior of the concretes and the mortars containing these fibers of cement substitution. We noted that the use of these fibers in the concretes in substitution of cement decreases its of compressive strength and flexural strength but to $10 \%$ of waste these strength remain acceptable.
\end{abstract}

\section{Introduction}

La recherche et l'expérience acquises ont permis l'introduction des fibres dans le domaine de la construction et qui rend la possibilité de produire des éléments minces, plus légers, plus résistants et moins, sujets à la fissuration par la simple addition d'une quantité de fibres [1]. Aujourd'hui, le respect de notre environnement et la sauvegarde des ressources naturelles obligent les autorités, et entreprises à encourager sérieusement la valorisation de ses déchets et l'utilisation de matériaux recyclés, en particulier dans le bâtiment [2].

\section{Essais sur mortiers}

Les mortiers sont réalisés conformément à la norme EN 196-1, se sont des éprouvettes prismatiques de $4 \times 4 \times 16 \mathrm{~cm}^{3}$.

\subsection{Matériaux utilisés:}

- Le ciment : le ciment utilisé est conforme aux normes algériennes NA 422 et NA 44, c'est le CEM I 42,5 (CPA).

- L'eau de gâchage : l'eau utilisée est l'eau potable de robinet de la ville de Bejaia (Algérie).

- Le sable : les sable utilisé est un sable normalisé (CEN EN 196-1), se sable est commercialisé en sac plastique de $1350 \pm 5 \mathrm{~g}$.
- Les fibres métalliques (rejet BCR) : se sont des fibres métalliques plates de 1 à $3 \mathrm{~cm}$ de longueur.

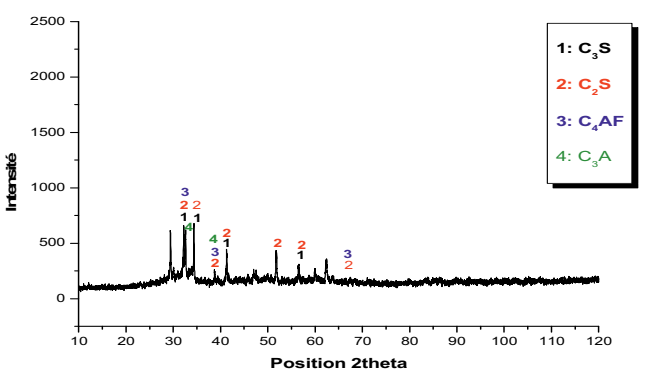

Fig. 1. DRX du ciment CPA.

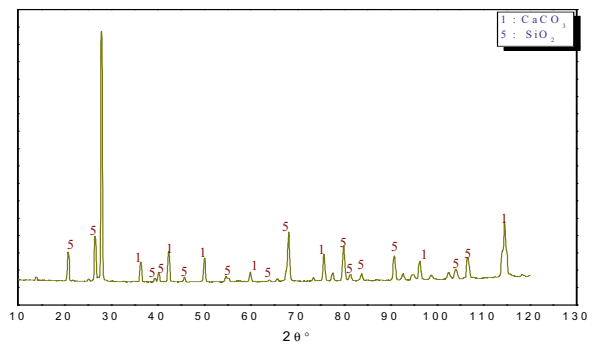

Fig. 2. DRX du Sable normalisé. 


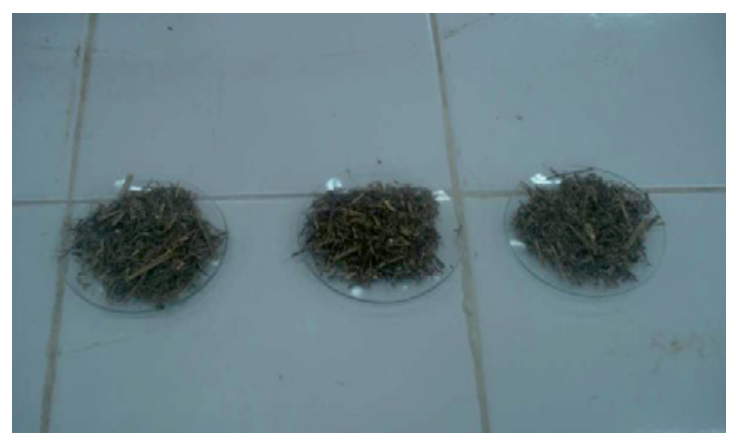

Fig. 3. Les fibres métalliques.

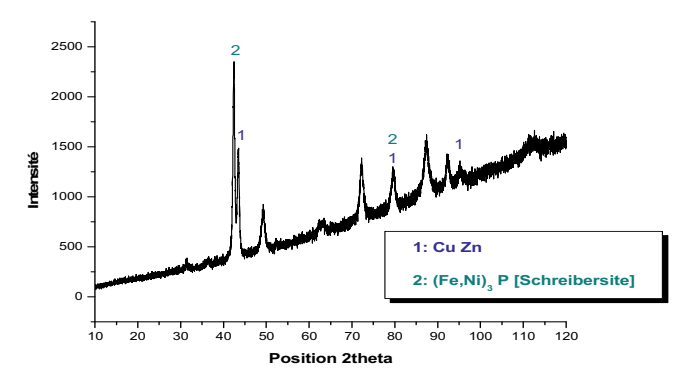

Fig. 4. Spectre obtenu par DRX pour les fibres.

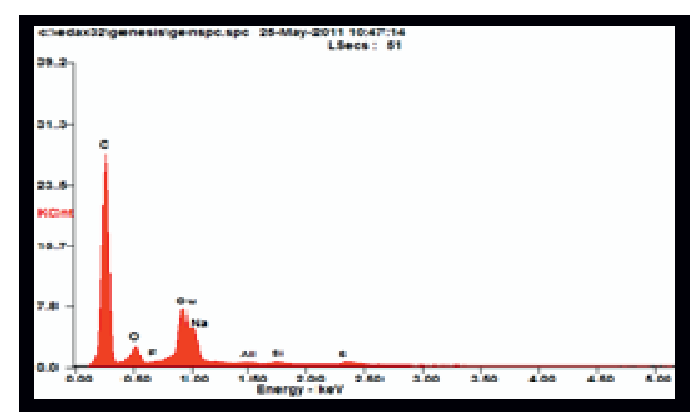

Fig. 5. Spectre EDX des fibres (le déchet) (par le MEB).

\subsection{Dosages et pesées:}

Dans le but de connaitre les effets des fibres sur les caractéristiques des mortiers, on utilise ces fibres à différents pourcentages en substitution de ciment, les quantités sont les suivantes :

Tableau 1. Dosage et pesées des constituants.

\begin{tabular}{|c|c|c|c|c|c|}
\hline $\begin{array}{c}\mathbf{N}^{\mathbf{0}} \mathbf{d e} \\
\mathbf{l a} \\
\text { série }\end{array}$ & $\begin{array}{c}\text { \% des } \\
\text { fibres }\end{array}$ & $\begin{array}{c}\text { Les } \\
\text { fibres } \\
(\mathbf{g})\end{array}$ & $\begin{array}{c}\text { Ciment } \\
(\mathbf{g})\end{array}$ & $\begin{array}{c}\text { Sable } \\
(\mathbf{g})\end{array}$ & $\begin{array}{c}\text { Eau } \\
(\mathbf{g})\end{array}$ \\
\hline M0 & 0 & 0 & 450 & 1350 & 225 \\
\hline M10 & 10 & 45 & 405 & 1350 & 225 \\
\hline M20 & 20 & 90 & 360 & 1350 & 225 \\
\hline M30 & 30 & 135 & 315 & 1350 & 225 \\
\hline
\end{tabular}

\subsection{Conservation des éprouvettes:}

a. Conservation des éprouvettes pour la résistance à la compression et pour la mesure du gonflement:

Une fois démoulées, marquées et éventuellement pesées, les éprouvettes sont conservées entièrement immergées dans l'eau distillée à $20^{\circ} \mathrm{C} \pm 1^{\circ} \mathrm{C}$, jusqu'au moment de la rupture et de la mesure du gonflement.

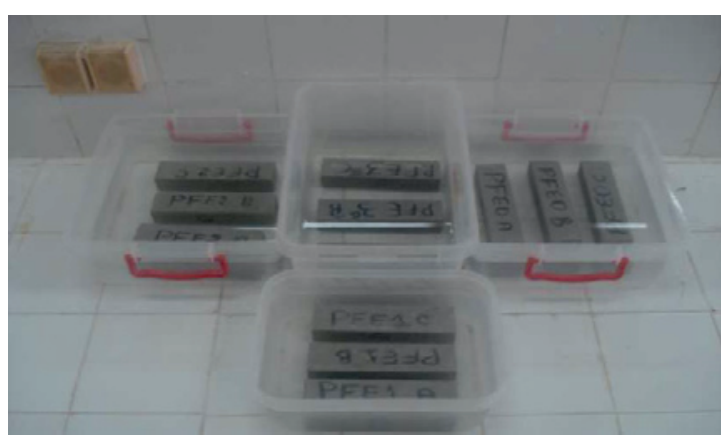

Fig. 6. Conservation des éprouvettes pour la résistance à la compression et pour la mesure du gonflement.

b. Conservation des éprouvettes pour la mesure du retrait:

La conservation des éprouvettes pour le retrait se fait à l'air libre sans.

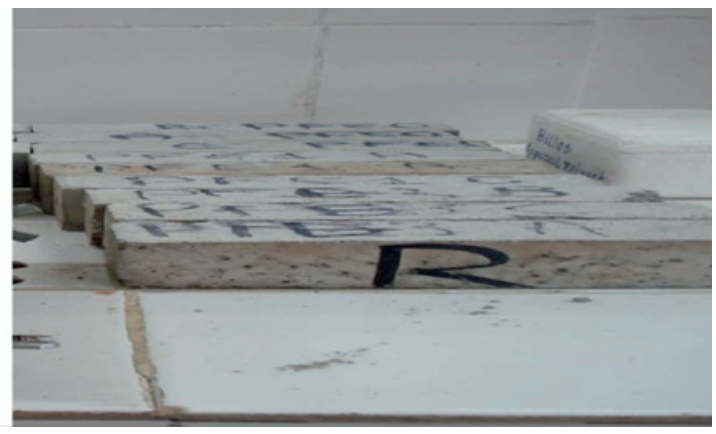

Fig. 7. Conservation des éprouvettes pour la mesure du retrait.

\subsection{Résultats des résistances à la compression et les résistances de traction par flexion:}

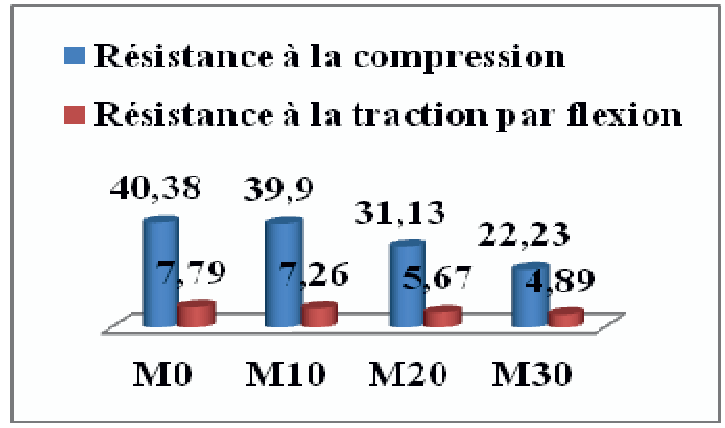

Fig. 8. Résultats des résistances mécaniques des mortiers $4 \times 4 \times 16 \mathrm{~cm}^{3}$ à 28 jours. 


\subsection{Résultats des mesures des variations dimensionnelles des éprouvettes de mortier :}

a. Mesure de retrait :

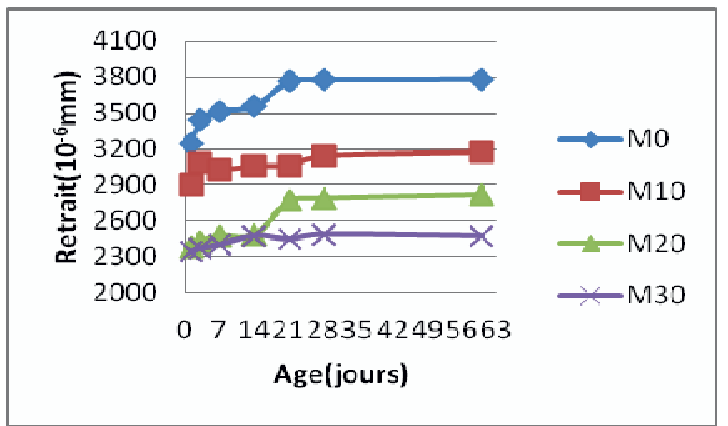

Fig. 9. Mesure de retrait jusqu'à $60 j$.

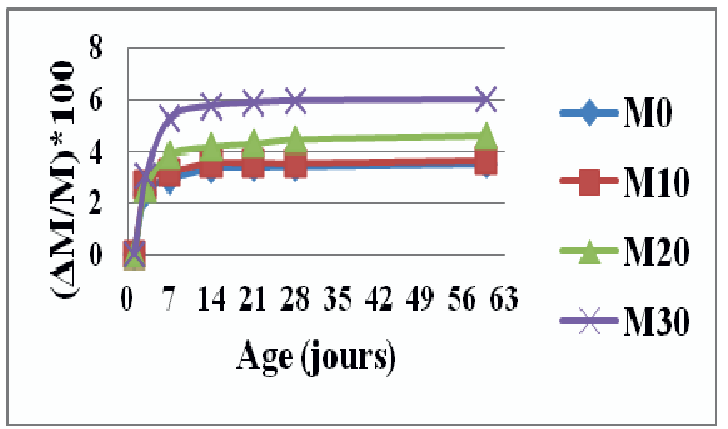

Fig. 10. Mesure de la variation de masse pendant le retrait jusqu'à $60 \mathrm{j}$.

b. Mesure du gonflement :

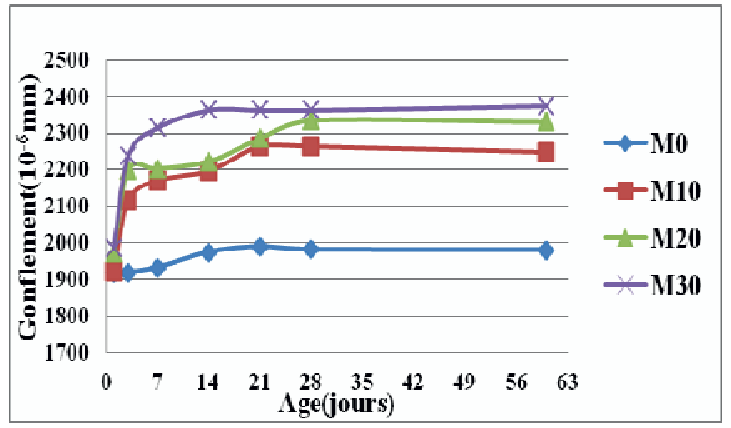

Fig. 11. Mesure du gonflement jusqu'à $60 j$.

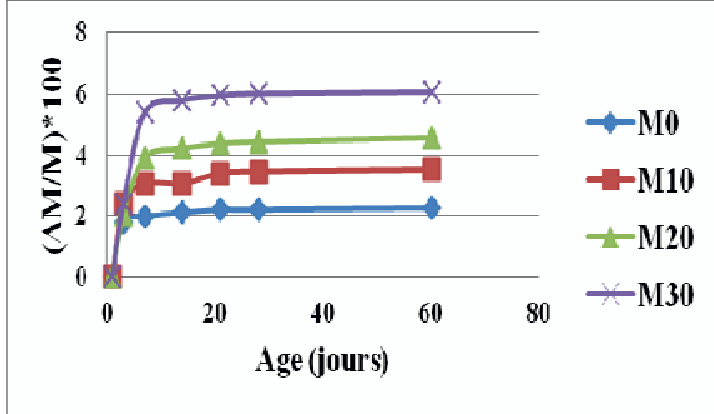

Fig. 12. Mesure de la variation de masse pendant le gonflement jusqu'à $60 \mathrm{j}$.

\section{Essais sur Bétons}

Les Bétons sont réalisés conformément à la norme NF-P $18-400$, se sont des éprouvettes cylindriques de $16 \times 32$ $\mathrm{cm}^{2}$. La méthode de formulation utilisée pour la détermination des dosages des différentes séries de bétons est celle de DREUX-GORISSE [3]

\subsection{Matériaux utilisés:}

- Le ciment: est le même ciment utilisé pour la confection des mortiers.

- L'eau de gâchage : est la même eau utilisée pour la confection des mortiers.

- Le sable : le sable utilisé est un sable 0/3 concassé de la carrière de BOUANDAS wilaya de Sétif (Algérie).

- Les graviers : les graviers utilisés sont des graviers $8 / 15$ et 15/25 concassés de la carrière de BOUANDAS.

- Les fibres métalliques (rejet BCR) : se sont les mêmes fibres utilisées pour la confection des mortiers.

Tableau 2. Analyses chimique du sable 0/3.

\begin{tabular}{|c|c|c|c|c|}
\hline $\begin{array}{c}\text { \% } \\
\text { insoluble }\end{array}$ & \% $\mathbf{C a C O}_{3}$ & \% Nacl & $\begin{array}{c}\text { \% } \\
\mathbf{C a S O}_{4}, \\
\mathbf{2 H}_{\mathbf{2}} \mathbf{O}\end{array}$ & $\begin{array}{c}\text { Teneur } \\
\text { en anions } \\
\text { de sulfate } \\
\text { SO4- }\end{array}$ \\
\hline 02.84 & 96.89 & Néant & Néant & Néant \\
\hline
\end{tabular}

Ce sable est un sable calcaire, et il n'est pas agressif aux bétons.

\subsection{Formulation des bétons:}

Nous allons établir toutes les courbes granulaires sur un graphique du type normalisé AFNOR (graphique semilogarithmique), qui comporte en abscisse les ouvertures des tamis et en ordonnées les tamisâts cumulés. 


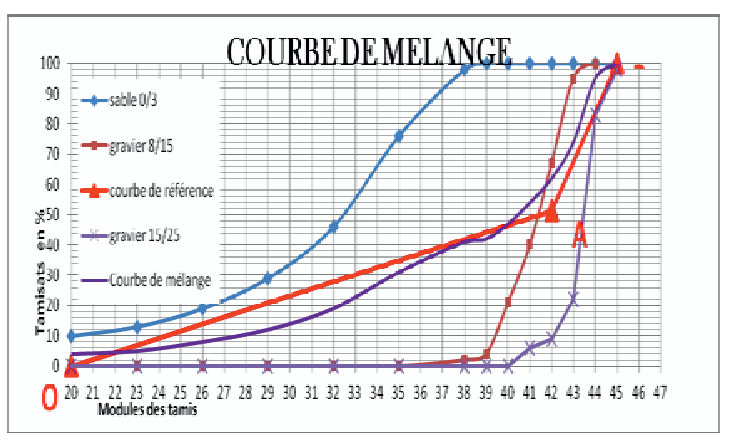

Fig. 13. Courbes du mélange selon la méthode de Dreux GORISSE.

\subsection{Détermination des dosages des constituants des bétons:}

Tableau 3. Dosages des constituants pour $1 \mathrm{~m}^{3}$ de béton.

\begin{tabular}{|c|c|c|c|}
\hline $\begin{array}{c}\text { Compo } \\
\text { sants }\end{array}$ & $\begin{array}{c}\% \\
\text { des } \\
\text { granulats }\end{array}$ & $\begin{array}{c}\text { Volume } \\
\text { absolu des } \\
\text { composants } \\
\text { (litres) } \\
\end{array}$ & $\begin{array}{c}\rho_{\mathrm{s}} \\
\left(\mathrm{g} / \mathrm{cm}^{3}\right)\end{array}$ \\
\hline Eau & - & 175.00 & 1 \\
\hline Ciment & - & 113.00 & 3.1 \\
\hline Sable $0 / 3$ & 34 & 232.56 & 2.72 \\
\hline Gravier 8/15 & 34 & 232.56 & 2.64 \\
\hline Gravier $15 / 25$ & 32 & 218.88 & 2.81 \\
\hline $\begin{array}{c}\text { Compo } \\
\text { sants }\end{array}$ & $\begin{array}{c}\text { Dosage des } \\
\text { composants } \\
\left(\mathbf{k g} / \mathbf{m}^{3}\right)\end{array}$ & $\begin{array}{c}\begin{array}{c}\text { Volumes } \\
\text { des }\end{array} \\
\text { composants } \\
\left(\mathbf{l} / \mathbf{m}^{3}\right) \\
\end{array}$ & $\underset{\left(\mathrm{g} / \mathrm{cm}^{3}\right)}{\mathbf{P}_{\mathbf{p}^{3}}}$ \\
\hline Eau & 175 & 175.00 & 1 \\
\hline Ciment & 350 & - & - \\
\hline Sable $0 / 3$ & 632.56 & 545.31 & 1.16 \\
\hline Gravier 8/15 & 613.95 & 479.64 & 1.28 \\
\hline Gravier $15 / 25$ & 615.05 & 445.68 & 1.38 \\
\hline
\end{tabular}

Tableau 4. Dosages des constituants des différentes gâchées de béton

\begin{tabular}{|c|c|c|c|}
\hline $\mathbf{N}^{\mathbf{0}}$ de série & $\begin{array}{c}\text { Gravier } \\
\mathbf{1 5 / 2 5} \mathbf{( k g )}\end{array}$ & $\begin{array}{c}\text { Gravier } \\
\mathbf{8 / 1 5}(\mathbf{k g})\end{array}$ & $\begin{array}{c}\text { Sable } \\
\mathbf{0 / 3} \mathbf{( k g )}\end{array}$ \\
\hline BT00 & 32.371 & 32.313 & 33.292 \\
\hline BT10 & 32.371 & 32.313 & 33.292 \\
\hline BT20 & 32.371 & 32.313 & 33.292 \\
\hline BT30 & 32.371 & 32.313 & 33.292 \\
\hline No de série & $\begin{array}{c}\text { Ciment } \\
\text { (kg) }\end{array}$ & $\begin{array}{c}\text { Fibres } \\
\text { métalliques } \\
\text { (kg) }\end{array}$ & $\begin{array}{c}\text { Eau } \\
(\mathbf{k g})\end{array}$ \\
\hline BT00 & 18.421 & 00.000 & 9.210 \\
\hline BT10 & 16.580 & 1.840 & 9.210 \\
\hline BT20 & 14.740 & 3.680 & 9.210 \\
\hline BT30 & 12.890 & 5.530 & 9.210 \\
\hline
\end{tabular}

\subsection{Conservation des éprouvettes de bétons:}

Les éprouvettes doivent rester dans les moules pendant $24 \mathrm{~h}$ et doivent être protégé contre les chocs, après démoulages elles doivent être entreposés dans l'eau à $20^{\circ} \mathrm{c} \pm 2^{\circ} \mathrm{c}$ jusqu' au moment de la rupture.

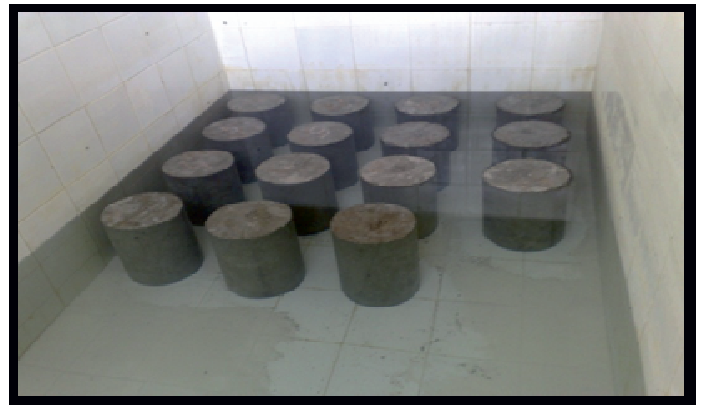

Fig. 14. Conservation des éprouvettes.

\subsection{Essai de compression (NF P 18-406) [4] :}

C'est un essai effectué sur un béton durci, il a pour but de connaitre la résistance à la compression du béton.

\subsection{Résultats des résistances à la compression:}

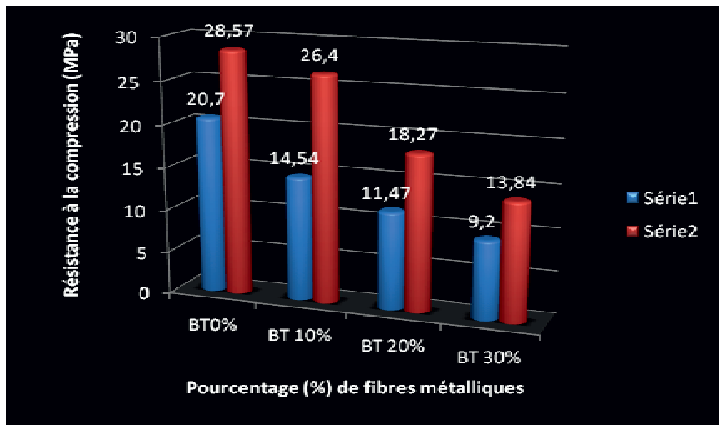

Fig. 15. Resistances à la compression des bétons à 7 et à 28 jours.

\section{Discussions et interprétations des résultats}

D'après les résultats obtenus on remarque que :

- Les résistances à la compression des mortiers et des bétons à l'âge de 28 jours diminuent au fur et à mesure d'augmenter le pourcentage des fibres métalliques, mais celles de $10 \%$ de fibres sont de bonnes résistances.

Cet abaissement est dû à la présence des fibres métalliques et à la diminution de la quantité du ciment dans le mélange donc l'augmentation du rapport $\mathrm{E} / \mathrm{C}$ qui fait diminuer la compacité ce qui influe sur la résistance, et c'est le cas pour les dosages à $20 \%$ et à $30 \%$ de fibres.

- On sait que la réaction d'hydratation s'accompagne d'une réduction de volume, appelée contraction. L'eau s'évapore d'un mortier conservé à l'air libre provoquant le retrait, qui est la conséquence de la perte d'eau libre, lorsque cette eau se retire du matériau, une contraction se produit automatiquement.

Les résultats présentés en figure .9. montrent un comportement de contraction des éprouvettes de mortiers à différents âges de mesure $(1 \mathrm{j}, 3 \mathrm{j}, 7 \mathrm{j}, 14 \mathrm{j}, 21 \mathrm{j}, 28 \mathrm{j}, 60 \mathrm{j})$, 
et à différents pourcentages de FMI $(0 \%, 10 \%, 20 \%$, $30 \%$ ), les changements volumiques se produisent après la prise. La variation du retrait des éprouvettes des mortiers à différents pourcentages sont presque du même ordre de grandeur mais celui du mortier témoin reste le plus grand, donc l'utilisation des FMI réduit le retrait et ça est dû à l'augmentation des pores capillaires qui emprisonne l'eau et l'empêche de s'évaporée, la diminution de la quantité du ciment provoque la diminution de son hydratation ce qui traduit la diminution $\mathrm{du}$ retrait. Pour tous les mortiers nous remarquons une évolution continue de retrait en fonction de l'âge, et que cette évolution commence a se stabilisée à partir du 28 jours.

- On sait que le retrait s'accompagne systématiquement d'une diminution ou d'une perte de masse, la figure .10. montre que la perte de masse augmente en fonction du temps, et commence à se stabiliser a partir de 28 jours pour tous les mortiers à différents pourcentages de fibres métalliques. Le mortier sans FMI a une perte de masse plus importante, cependant il a plus d'évaporation d'eau, se qui est dû à une hydratation plus importante du ciment. - La figure .11. montre que le gonflement augmente en fonction $\mathrm{du}$ temps et $\mathrm{du}$ pourcentage des fibres métalliques en substitution de quantité du ciment, et il commence a se stabilisé à partir de 28 jours. L'augmentation du gonflement s'explique par la présence d'une quantité d'eau importante dans les pores crée par le manque d'hydratation du ciment et l'ajout des fibres métalliques.

- La figure .12. montre que la variation des masses pour le gonflement est proportionnelle à celle du gonflement lui-même, l'augmentation de cette masse est due à la quantité d'eau absorbée par les éprouvettes du mortier.

\section{Conclusion}

D'après les résultats obtenus sur l'introduction des fibres métalliques en substitution du ciment on conclu que :

Les fibres métalliques peuvent être utilisées comme remplaçant du ciment a $10 \%$, après avoir présenté une résistance à la compression acceptable ( $>25 \mathrm{MPa})$, donc la formulation à $10 \%$ de fibres est intéressante du faite qu'elle présente une bonne résistance à la compression et à la flexion, un retrait et un gonflement acceptables qui sont proches de ceux de $0 \%$ (mortier témoin). C'est un gain économique concernant le ciment, et un plus environnemental permettant de dégagé des espaces de stockage au niveau de l'usine.

Donc ces fibres métalliques de BCR peuvent être utilisées dans les bétons de structures.

\section{References}

1. A. Belfarrag, Valorisation des fibres métalliques issues des déchets pneumatiques dans les bétons. Thèse de Magister, Université de Ouargla (2006).

2. F. Boukhatem, M.A. Fergani, Etude expérimentale des débris de maçonnerie dans les mortiers. Thèse d'ingéniorat, Ecole National Polytechnique. (2006)

3. G.DREUX et J.FESTA, Nouveau guide du béton, Edition EYROLLES, (1997)

4. R. Dupain, R. Lanchon, J.C. Arroman, Granulats, Sols, Ciments et Bétons, Edition Casteila (2004) 